\title{
Lung microbiome dynamics in COPD exacerbations
}

\author{
Zhang Wang ${ }^{1,7}$, Mona Bafadhel $^{2,7}$, Koirobi Haldar ${ }^{3,6}$, Aaron Spivak $^{1}$, \\ David Mayhew ${ }^{1}$, Bruce E. Miller ${ }^{4}$, Ruth Tal-Singer ${ }^{4}$, Sebastian L. Johnston ${ }^{5}$, \\ Mohammadali Yavari Ramsheh ${ }^{3}$, Michael R. Barer ${ }^{3}$, Christopher E. Brightling ${ }^{3,6,8}$ \\ and James R. Brown ${ }^{1,8}$
}

\begin{abstract}
Affiliations: ${ }^{1}$ Computational Biology, Target Sciences, GSK R\&D, Collegeville, PA, USA. ${ }^{2}$ Respiratory Medicine Unit, Nuffield Department of Clinical Medicine, University of Oxford, Oxford, UK. ${ }^{3}$ Institute for Lung Health, National Institute for Health Research Respiratory Biomedical Research Unit, Dept of Infection, Immunity and Inflammation, University of Leicester, Leicester, UK. ${ }^{4}$ Respiratory Therapy Area Unit, GSK R\&D, King of Prussia, PA, USA. ${ }^{5}$ Airway Disease Infection Section, National Heart and Lung Institute, Imperial College London, London, UK. ${ }^{6}$ Dept of Health Sciences, University of Leicester, Leicester, UK. ${ }^{7}$ These authors contributed equally. ${ }^{8}$ Both authors contributed equally.
\end{abstract}

Correspondence: Christopher E. Brightling, Institute for Lung Health, Clinical Sciences Wing, University Hospitals of Leicester, Leicester, LE3 9QP, UK. E-mail: ceb17aleicester.ac.uk

ABSTRACT Increasing evidence suggests that the lung microbiome plays an important role in chronic obstructive pulmonary disease (COPD) severity. However, the dynamics of the lung microbiome during COPD exacerbations and its potential role in disease aetiology remain poorly understood.

We completed a longitudinal $16 \mathrm{~S}$ ribosomal RNA survey of the lung microbiome on 476 sputum samples collected from 87 subjects with COPD at four visits defined as stable state, exacerbation, 2 weeks post-therapy and 6 weeks recovery.

Our analysis revealed a dynamic lung microbiota where changes appeared to be associated with exacerbation events and indicative of specific exacerbation phenotypes. Antibiotic and steroid treatments appear to have differential effects on the lung microbiome. We depict a microbial interaction network for the lung microbiome and suggest that perturbation of a few bacterial operational taxonomic units, in particular Haemophilus spp., could greatly impact the overall microbial community structure. Furthermore, several serum and sputum biomarkers, in particular sputum interleukin-8, appear to be highly correlated with the structure and diversity of the microbiome.

Our study furthers the understanding of lung microbiome dynamics in COPD patients and highlights its potential as a biomarker, and possibly a target, for future respiratory therapeutics.

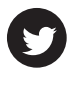

@ERSpublications

Lung microbiome changes are associated with COPD exacerbation events and implicated in host inflammatory responses http://ow.ly/Wamjx

For editorial comment see Eur Respir J 2016; 47: 1034-1036 [DOI: 10.1183/13993003.00270-2016].

This article has supplementary material available from erj.ersjournals.com

Received: Aug 242015 | Accepted after revision: Dec 062015 | First published online: Feb 252016

Support statement: The study was sponsored by University Hospitals of Leicester National Health Service (NHS) Trust. Study cohort BEAT-COPD (Biomarkers to Target Antibiotic and Systemic Corticosteroid Therapy in COPD Exacerbations) was co-funded by the Medical Research Council (UK) and AstraZeneca, with microbiome analysis funded by GlaxoSmithKline (GSK). C.E. Brightling and M. Bafadhel were funded by the National Institute for Health Research (NIHR) with additional support from the Leicester NIHR Respiratory Biomedical Research Unit. Z. Wang is supported by the GSK Early Talent Postdoctoral Fellowship Program. The views expressed are those of the authors and not necessarily those of the NHS, the NIHR or the Department of Health.

Clinical trial: This study is registered at www.isrctn.com with identifier number 92422949.

Conflict of interest: Disclosures can be found alongside the online version of this article at erj.ersjournals.com

Copyright OERS 2016 


\section{Introduction}

Chronic obstructive pulmonary disease (COPD), one of the most prevalent respiratory diseases, is characterised by persistent symptoms and impaired lung function as a consequence of airway inflammation, small airway obliteration and alveolar destruction [1-3]. Acute exacerbations of COPD are sudden worsening of symptoms in which bacterial colonisation is one major aetiological factor [4-7]. However, the dynamics of bacterial ecology during exacerbations and its role in disease pathogenesis remain poorly understood.

Advances in next-generation DNA sequencing have provided an opportunity to study the lung microbiome in health and disease. Studies using culture-independent techniques such as PCR amplification and sequencing of the 16S ribosomal (r)RNA gene have characterised a distinct bacterial community in the airway of COPD patients compared to healthy subjects and suggest that changes in the lung microbiota could be associated with enhanced airway inflammation and disease progression [8-11]. However, most lung microbiome studies to date have involved relatively small cohorts of subjects with limited longitudinal sampling and concurrent clinical information.

We hypothesise that incorporating the lung microbiome profile from larger and better-characterised patient cohorts may improve our mechanistic understanding of COPD aetiology as well as provide additional prognostic and therapeutic signatures. Here, we performed a longitudinal 16S rRNA-based microbiome survey on 476 sputum samples collected from 87 subjects with COPD from BEAT-COPD (Biomarkers to Target Antibiotic and Systemic Corticosteroid Therapy in COPD Exacerbations) [12, 13], which, to our knowledge, is the largest well-characterised COPD lung microbiome cohort to date. We found that changes in the lung microbiome were associated with specific clinical and biochemical characteristics of COPD, thereby providing further insights into the relationships among the lung microbial community, host inflammatory responses and disease pathogenesis.

\section{Methods}

\section{Subjects and samples}

Sputum samples from COPD subjects were longitudinally collected at four visit types: stable state (defined as being 8 weeks free from an exacerbation), exacerbation (defined according to Anthonisen criteria [14] and/or healthcare utilisation [15]), 2 weeks post-therapy and at recovery (6 weeks post-exacerbation visit). Exacerbations were treated with oral corticosteroids and antibiotics according to guidelines [16] or study design [13]. The online supplementary material provides additional information on methods, study subjects, DNA sequencing, reagent controls and biostatistical analyses.

\section{Microbiome analysis}

Bacterial genomic DNA was extracted from sputum samples using the Qiagen DNA Mini kit (Qiagen, Valencia, CA, USA) as per manufacturer's protocol, and the V3-V5 hypervariable regions of the 16S rRNA gene were PCR-amplified with the appropriate controls against reagent contamination. Amplified DNA fragments were pyrosequenced using the 454 Genome Sequencer FLX platform (454 Life Sciences; Roche Diagnostics, Burgess Hill, UK). Sequencing reads were processed using QIIME (quantitative insights into microbial ecology) pipeline version 1.7 [17]. Stringent criteria were used to remove low-quality and chimeric reads. The remaining reads were subject to open reference operational taxonomic unit (OTU) picking (97\% identity cut-off). Sequence data are deposited at the National Center for Biotechnology Information Sequence Read Archive (SRP065072).

\section{Statistical analyses}

Briefly, exacerbation phenotypes were defined using microbiological and clinical criteria as established previously [12]. Phenotypes of 28 exacerbations samples were undetermined due to missing data. Partial least squares discriminant analysis (PLS-DA), receiver operating characteristic curve reconstruction and network analysis were performed on exacerbation phenotypes and microbiota and/or clinical data. A general linear mixed model (GLMM) was constructed between clinical variables and four measures of $\alpha$ diversity (microbial diversity within a sample): OTU richness, Shannon's H, chaol and Faith's phylogenetic diversity. To identify clinical predictors of $\beta$ diversity (microbial composition dissimilarity between samples), canonical correspondence analysis (CCA) was performed on clinical variables and the relative abundance of taxa at the phylum, genus and OTU levels. Biomarker factors were identified using principal component analysis (PCA). The false discovery rate method was used to adjust (adj.) p-values for multiple tests [18].

\section{Results}

\section{Lung microbiome profiles}

Sequential sputum samples from 87 subjects were obtained at stable state, exacerbation, 2 weeks post-therapy and 6 weeks recovery (figure 1). At exacerbation, subjects were treated with antibiotics, oral corticosteroids or a combination of both according to guidelines [16] or study design [13]. All samples at 


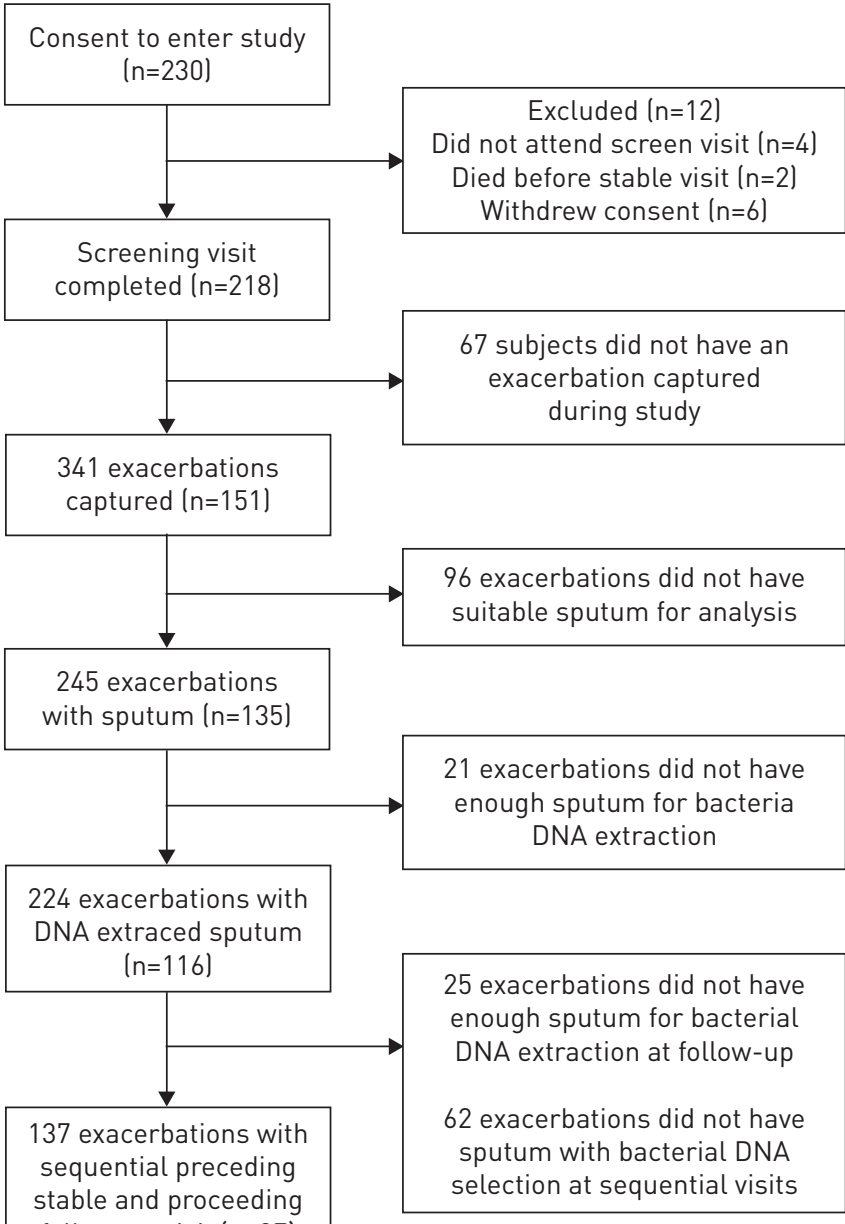

FIGURE 1 Flow diagram for BEAT-COPD (Biomarkers to Target Antibiotic and Systemic Corticosteroid Therapy in COPD Exacerbations) follow-up visit $(n=87)$

subject enrolment.

exacerbation onset were obtained prior to initiation of treatment. Tables 1 and 2 show the clinical characteristics of the subjects. Sputum and serum mediator data were collected in stable and exacerbation samples of a proportion of subjects $(n=54)$, as reported previously (hereafter described as group I subjects) [12]. 4500748 DNA sequencing reads were generated after demultiplexing and quality control filtering. A rarefaction depth of 1666 reads per sample was selected based on jack-knifed principal coordinate analysis (PCoA) re-sampling analysis (online supplementary figure S1). 1193 OTUs were identified across 476 samples.

Based on overall phyla composition, samples clustered into one of three groups: Proteobacteria, Firmicutes or Bacteroidetes subgroups (online supplementary figure S2). $\sim 98.4 \%$ of sequences belonged to one of four phyla: Firmicutes (51.4\%), Proteobacteria (35.9\%), Actinobacteria (6.5\%) or Bacteroidetes (4.6\%). Of the 366 genera identified, the most abundant were Streptococcus (41.1\%), Haemophilus (18.9\%), Moraxella (5.6\%) and Pseudomonas (4.4\%), all of which are typical members of the lung microbiota [24]. The genera Streptococcus (prevalence 95.0\%), Haemophilus (94.7\%), Rothia (94.1\%), Veillonella (93.2\%) and Prevotella $(90.3 \%)$ were highly prevalent across all visits (table 3 ). There was a significantly greater inter-subject variation in microbiome community at the same visit (weighted UniFrac \pm SD $0.387 \pm 0.186$ ) compared with temporal variation within each subject (weighted UniFrac \pm SD $0.272 \pm 0.181, p=2.2 \mathrm{e}-16$ using $\mathrm{t}$-test), suggesting that for our cohort the lung microbiome was relatively stable over time [8].

\section{Microbiome shifts during exacerbations}

Previous reports have emphasised the importance of bacteria in COPD exacerbations [19, 20]. Our results indicate an overall reduced $\alpha$ diversity (microbial diversity within a sample) with a small and nonsignificant microbial composition shift toward an increase in the relative abundance of Proteobacteria (adj. $\mathrm{p}=0.42$ using paired t-test) and a decrease in Firmicutes (adj. $\mathrm{p}=0.73$ using paired t-test) during exacerbations compared to stable samples (figure 2a). Moraxella showed the greatest change during exacerbations, averaging an increase of relative abundance by $5 \%$ (adj. $\mathrm{p}=0.22$ using paired t-test; figure $2 \mathrm{a}$ and $\mathrm{b}$ ). This was followed by a decrease in Streptococcus (3.8\%, adj. $\mathrm{p}=0.58$ using paired t-test) and an increase in 
TABLE 1 Major clinical characteristics of subjects at baseline

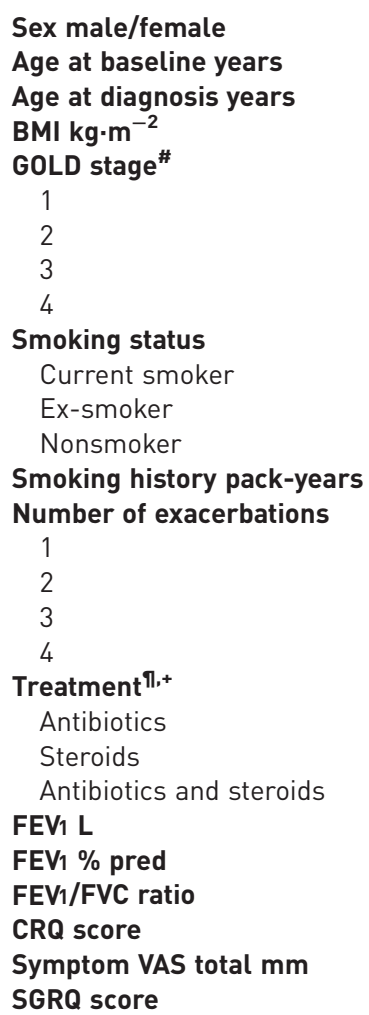

$65 / 22$

$68(45-87)$

$61(30-83)$

$26.40(16.67-38.19)$

1
35
32
19
37
48
2
$50(6-158)$
46
31
9
1

21
8
65
$1.3 \pm 0.1$
$47.4 \pm 2.0$
$46.7 \pm 1.4$
$16.2 \pm 0.5$
$159.6 \pm 8.5$
$52.9 \pm 1.9$

Data are presented as $\mathrm{n}$, mean (range) or mean \pm SEM. BMI: body mass index; GOLD: Global Initiative for Chronic Obstructive Lung Disease; FEV1: forced expiratory volume in 1s; FVC: forced vital capacity; CRQ: Chronic Respiratory Disease Questionnaire; VAS: visual analogue scale; SGRQ: St George's Respiratory Questionnaire. \#: baseline visits prior to the stable visits for sputum collection; ": treatments administered for exacerbations; assessments at exacerbation were prior to initiation of therapy; ${ }^{+}$: numbers represent exacerbation events, thus include subjects with more than one exacerbation.

TABLE 2 Major clinical characteristics of subjects over four visits

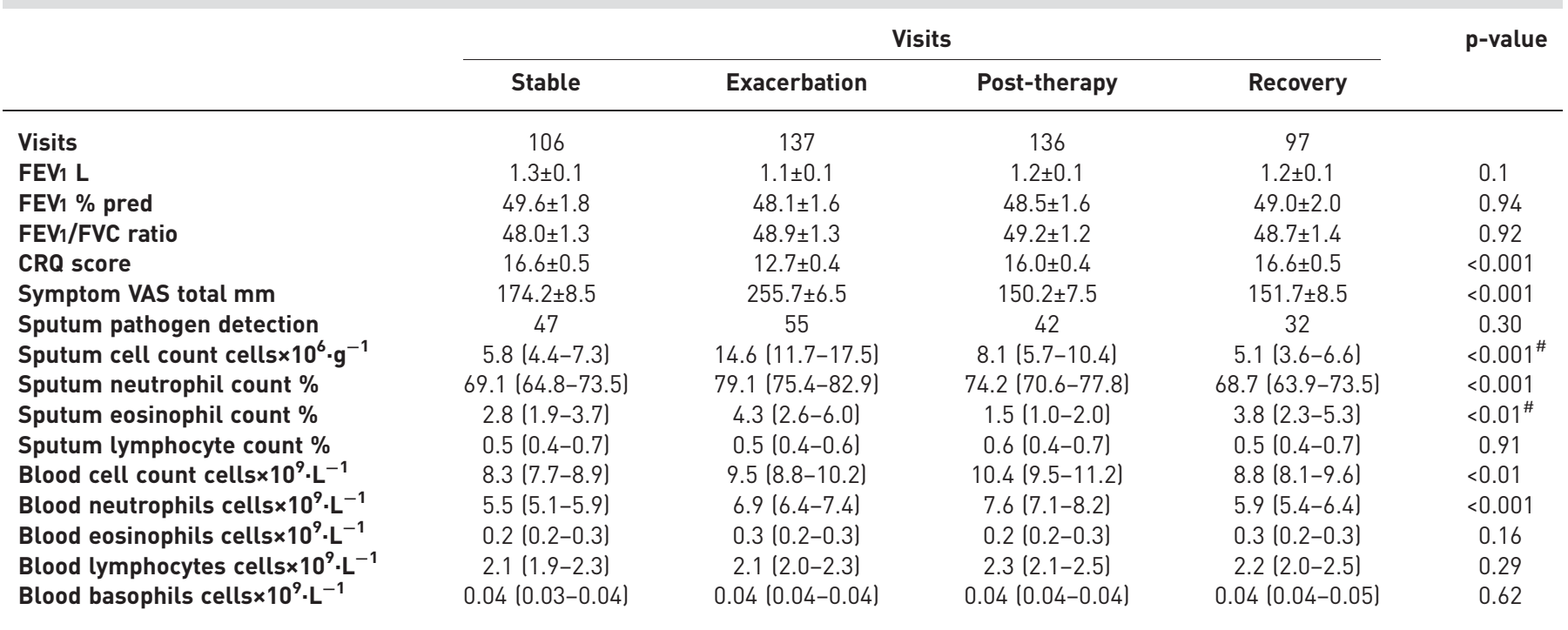

Data are presented as $\mathrm{n}$, mean \pm SEM or mean $(95 \% \mathrm{CI})$. FEV1: forced expiratory volume in 1s; FVC: forced vital capacity; CRQ: Chronic Respiratory Disease Questionnaire; VAS: visual analogue score. ${ }^{\#}$ : these variables were log transformed for statistical analysis. 
TABLE 3 The prevalence (P) and average relative abundance (RA) of predominant operational taxonomic units (OTUs) (average relative abundance $>1 \%$ ) in the lung microbiome

\begin{tabular}{|c|c|c|c|c|c|c|c|c|c|c|c|}
\hline OTU ID & Species/subspecies & \multicolumn{2}{|c|}{ All } & \multicolumn{2}{|c|}{ Stable } & \multicolumn{2}{|c|}{ Exacerbation } & \multicolumn{2}{|c|}{ Post-therapy } & \multicolumn{2}{|c|}{ Recovery } \\
\hline Visits $\mathrm{n}$ & & \multicolumn{2}{|c|}{476} & \multicolumn{2}{|c|}{106} & \multicolumn{2}{|c|}{137} & \multicolumn{2}{|c|}{136} & \multicolumn{2}{|c|}{97} \\
\hline 4445466 & Streptococcus spp. & 44.7 & 10.1 & 47.2 & 12.1 & 45.3 & 8.9 & 46.3 & 10.1 & 39.2 & 9.5 \\
\hline 509773 & Streptococcus spp. & 78.4 & 6.6 & 85.8 & 7.3 & 80.3 & 6.3 & 71.3 & 6.2 & 77.3 & 6.9 \\
\hline 1059655 & Streptococcus spp. & 31.5 & 5.2 & 30.2 & 5.2 & 33.6 & 5.4 & 30.9 & 4.9 & 30.9 & 5.6 \\
\hline 956702 & Haemophilus spp. & 25.2 & 5.1 & 33.0 & 6.5 & 24.1 & 5.1 & 23.5 & 5.4 & 20.6 & 3.0 \\
\hline 4385138 & Haemophilus spp. & 23.5 & 1.4 & 25.5 & 0.8 & 27.0 & 1.9 & 22.1 & 0.9 & 18.6 & 1.9 \\
\hline 861881 & Moraxella spp. & 46.2 & 5.6 & 45.3 & 5.0 & 57.7 & 10.0 & 41.9 & 3.4 & 37.1 & 3.3 \\
\hline 269930 & Pseudomonas veronii & 33.8 & 2.4 & 42.5 & 3.0 & 32.8 & 2.6 & 32.4 & 2.2 & 27.8 & 1.8 \\
\hline 269901 & Pseudomonas spp. & 12.8 & 1.6 & 14.2 & 1.8 & 12.4 & 1.2 & 14.7 & 1.4 & 9.3 & 2.4 \\
\hline 342427 & Veillonella dispar & 93.3 & 2.7 & 91.5 & 2.4 & 93.4 & 2.2 & 94.9 & 3.2 & 92.8 & 3.0 \\
\hline 257492 & Granulicatella spp. & 83.8 & 1.4 & 86.8 & 1.4 & 85.4 & 1.2 & 80.9 & 1.3 & 82.5 & 1.7 \\
\hline 4307391 & Prevotella melaninogenica & 39.1 & 1.1 & 41.5 & 1.1 & 39.4 & 1.2 & 40.4 & 1.2 & 34.0 & 0.8 \\
\hline
\end{tabular}

Data are presented as \%, unless otherwise stated. The OTUs were firstly grouped by their genera and then ranked by their RAs.

Haemophilus (3.0\%, adj. $\mathrm{p}=0.57$ using paired $\mathrm{t}$-test). Although these changes were not statistically significant in comparisons of paired stable and exacerbation samples, a significant increase in Moraxella was observed when comparing exacerbation versus all nonexacerbation samples (adj. $\mathrm{p}=0.022$ using t-test).

Further taxonomic breakdown reveals that $95.6 \%$ of Moraxella DNA sequence reads corresponded to a single OTU (861881) which has $100 \%$ sequence identity to the same 16S rRNA segment from the respiratory pathogen Moraxella catarrhalis [21]. A significant positive correlation was also found between the relative abundance of OTU 861881 and the bacterial load of $M$. catarrhalis as measured by quantitative PCR (online supplementary figure S3). The abundance of Moraxella was most significantly correlated with two host factors, the percentages of sputum neutrophils (adj. $\mathrm{p}=0.002, \mathrm{r}=0.18$ ) and sputum macrophages (adj. p=0.005, r=-0.19).

Despite an overall increase of Moraxella during exacerbations across the entire dataset, comparison of paired samples from the same subjects revealed some heterogeneity in Moraxella changes (figure 2c). Increase in the relative abundance of Moraxella during exacerbations was observed in 36 out of the 87 subjects due to a potential acquisition of a new Moraxella OTU in 23 subjects in whom it was undetectable in the stable samples and a dramatic outgrowth of a pre-existing Moraxella species by an average of 268-fold (ranging from 2.3- to 1412-fold) in another 13 subjects.

\section{Microbiome discriminates between bacterial and eosinophilic exacerbations}

Exacerbation phenotypes were defined as either bacterial ( $\mathrm{n}=33$ exacerbation samples), eosinophilic $(\mathrm{n}=19)$, viral $(\mathrm{n}=15)$, bacterial/eosinophilic combination $(\mathrm{n}=3)$, bacterial/viral combination $(\mathrm{n}=12)$ or pauci-inflammatory $(\mathrm{n}=27)$ using previously published biological criteria [12]. Distinct microbiome profiles at both the phylum and genus levels were observed during exacerbations among subjects across different phenotypes (figure 3a). Differences were most pronounced between bacterial and eosinophilic exacerbations, which were more dissimilar from each other in composition than either was to the other subgroups. In particular, there was a significant decrease of $\alpha$ diversity ( $\mathrm{p}=0.04 \mathrm{using} \mathrm{t}$-test) and Firmicutes (adj. $\mathrm{p}=6.3 \mathrm{e}-5$ using t-test) and an increase of Proteobacteria (adj. $\mathrm{p}=2 \mathrm{e}-4$ using t-test) in the bacterial subgroup compared to the eosinophilic subgroup (figure 3a). At the genus level, this corresponded to a significant decrease in Streptococcus (adj. $\mathrm{p}=0.002$ using t-test) and increase in Haemophilus (adj. $\mathrm{p}=0.008$ using t-test) in the bacterial subgroup. In addition, a notably decreased Proteobacteria:Firmicutes ratio was found in eosinophilic subgroups during exacerbations, in sharp contrast to all other subgroups (online supplementary figure S4). Furthermore, individual exacerbation samples in the bacterial and eosinophilic subgroups were relatively distinct from each other in both PCoA 

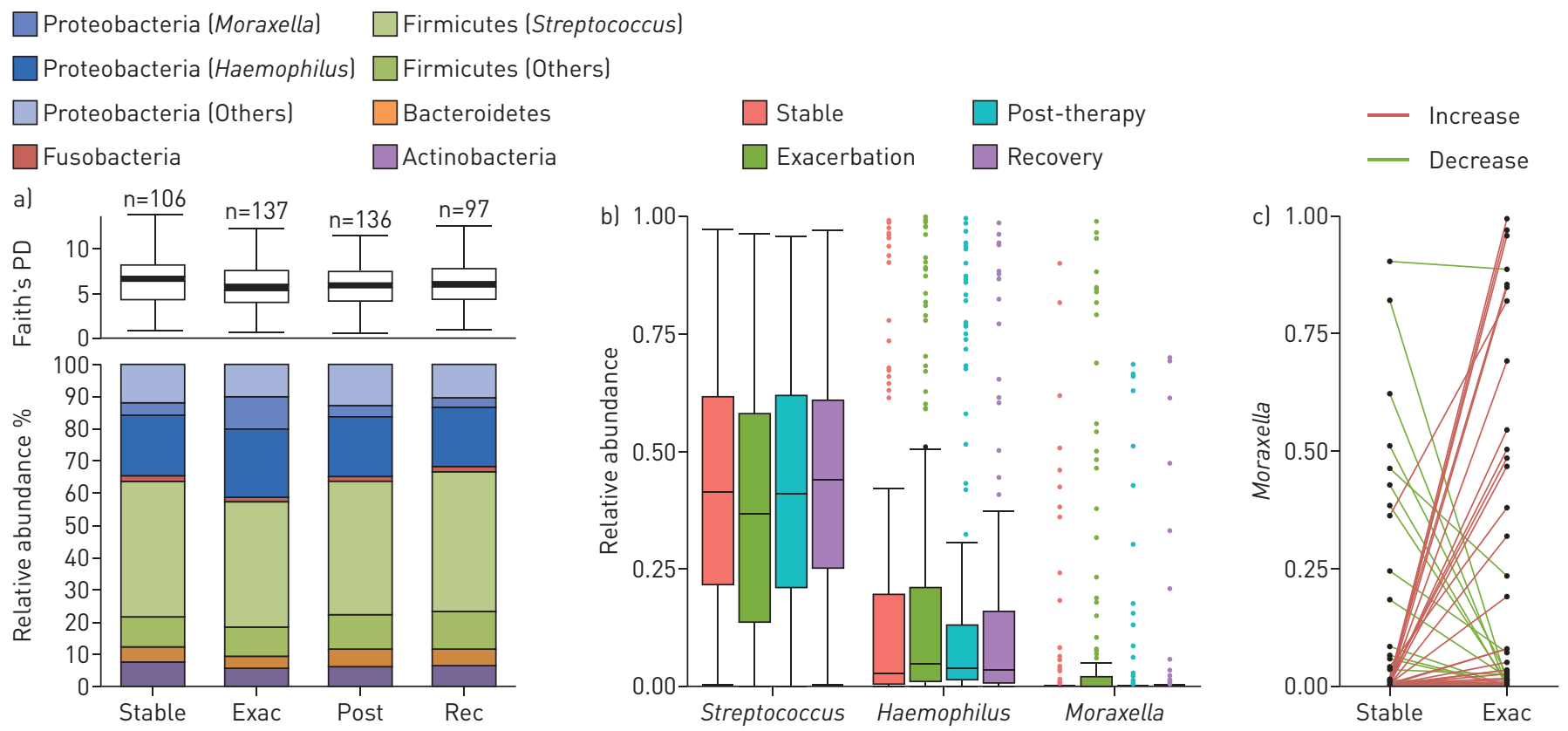

FIGURE 2 Microbiome shifts during exacerbations. a) $\alpha$ diversity (Faith's phylogenetic diversity (PD)) and composition of major taxonomic groups at both phylum and genus levels in samples collected across the four visit types: stable, exacerbation (Exac), post-therapy (Post) and recovery (Rec). b) Box and whisker plots showing the relative abundances of Streptococcus, Haemophilus and Moraxella in samples collected across the four visits. c) Heterogeneity in Moraxella changes among individuals. Lines connect paired stable and exacerbation samples collected in the same visit series from the same subjects, and were coloured by increase or decrease of Moraxella during exacerbations. Only paired stable and exacerbation samples were included.

(figure 3b) and unweighted pair group method with arithmetic mean clustering analyses (figure 3c). However, PLS-DA showed only a modest improvement in the prediction of bacterial and eosinophilic exacerbation events using combined clinical and microbiome data versus clinical data alone (figure and online supplementary table S1).

Oral corticosteroids and antibiotics have different effects on the lung microbiome

Since antibiotics and steroids can alter the lung microbiome in COPD patients [8], we investigated the changes in the microbiome resulting from these treatments. In agreement with HuANG et al. [8], we found a decreased microbial $\alpha$ diversity with an increase of Proteobacteria over Firmicutes in subjects treated with corticosteroids alone (figure 4). At the genus level, this corresponded to a decrease of Streptococcus and an increase of Haemophilus and Moraxella. An opposite trend in both $\alpha$ diversity and microbial composition changes was observed for subjects treated with antibiotics (with or without steroids). The effects of different treatments were further maintained from post-therapy to recovery, indicating a prolonged effect of treatment on the microbiome.

\section{Network analysis reveals potential microbiota interactions}

To gain insight into the interaction between bacterial OTUs in the lung microbiome, we performed an OTU network analysis using CoNet (co-occurrence network interference; http://psbweb05.psb.ugent.be/ conet/index.php) [22]. Examination of the microbial network revealed that it was predominated by a few "hub" OTUs that were highly connected with multiple other nodes (figure 5a and online supplementary table S2). For example, the OTU with the highest degree of connectivity in the network was OTU 240755 (Haemophilus spp.), which had a co-exclusive relationship with 33 other OTUs. This was followed by OTU 861881 (Moraxella spp.), OTU 956702 (Haemophilus spp.) and OTU 4445466 (Streptococcus spp.), all of which had numerous negative connections with other members of the microbiota. Consequently, abundance increases of OTU 240755, OTU 861881 and OTU 956702 were all associated with a significant decrease in microbial $\alpha$ diversity (Shannon's H versus OTU 240755 adj. $\mathrm{p}=1.6 \mathrm{e}-21, \mathrm{r}=-0.43$; OTU 861881 adj. $\mathrm{p}=8.8 \mathrm{e}-8, \mathrm{r}=-0.24$; and OTU 956702 adj. $\mathrm{p}=1.1 \mathrm{e}-9, \mathrm{r}=-0.28)$. Other than co-exclusions, co-existence patterns were also observed among bacterial species, such as five OTUs showing a strong mutual cooperative relationship in a tightly connected subgraph (figure 5a). 

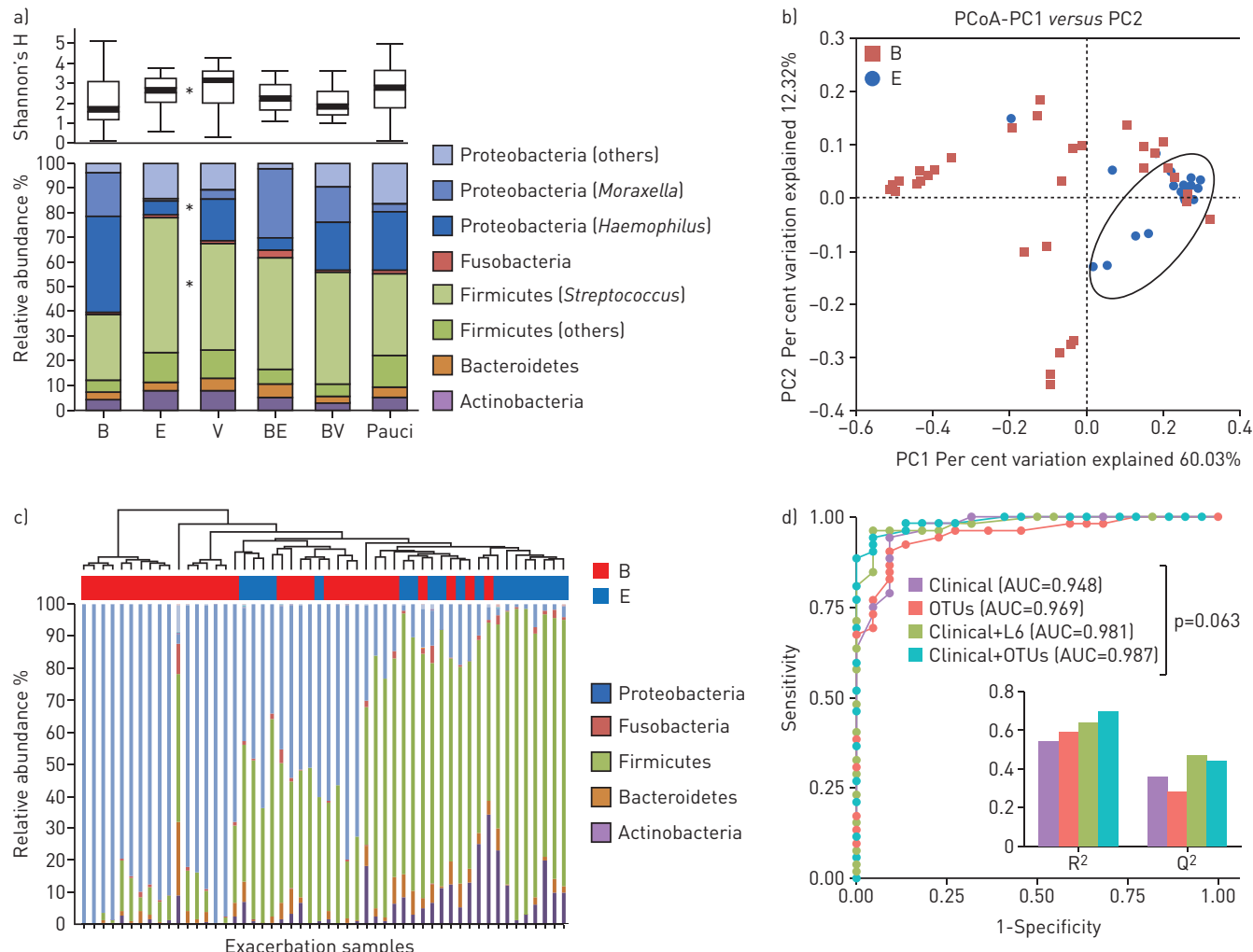

FIGURE 3 The microbiome discriminates bacterial and eosinophilic exacerbations. a) $\alpha$ diversity (Shannon's H) and composition of major taxonomic groups at both phylum and genus levels in exacerbation samples with different exacerbation phenotypes. The number of samples is indicated for each subgroup in the bar chart. b) Principal coordinate analysis (PCOA) and c) unweighted pair group method with arithmetic mean clustering show distinct clustering of samples in bacterial and eosinophilic subgroups. d) Partial least squares discriminant analysis classification of bacterial and eosinophilic exacerbations using clinical, microbiome and their combined variables at both phylum (L6) and operational taxonomic unit (OTU) levels. The models were evaluated in terms of area under the curve $(A U C), R^{2}$ and $Q^{2}$ scores. B: bacterial; V: viral; E: eosinophilic; $\mathrm{BE}$ : bacterial and eosinophilic; BV: bacterial and viral; and Pauci: pauci-inflammatory. *: $p<0.05$.

\section{Sputum chemokine (C-X-C motif) ligand 8/interleukin-8 as an indicator of community structure and diversity}

We applied an expanded network analysis which included both bacterial OTUs and clinical variables to investigate potential human host interactions with the lung microbiome. A set of 66 clinical variables were selected after exclusion of strongly mutually correlated variables. Several sputum mediators, including sputum interleukin (IL)-8/chemokine (C-X-C motif) ligand (CXCL)8, matrix metalloproteinase (MMP)-7 and MMP-8 appeared as highly connected nodes in the network (figure $5 \mathrm{~b}$ and online supplementary table S3). Among them, sputum CXCL8/IL-8 had the highest degree of microbiota connectivity with a significant negative correlation to 15 OTUs.

Sputum CXCL8/IL-8 also had a significant negative correlation with microbiome $\alpha$ diversity according to GLMM analysis ( $p<0.05$ using GLMM; table 4 and online supplementary figure S5). This finding was supported by PCA analysis where $\alpha$ diversity was negatively correlated with biomarker bactor 8 (or principal component 8 ) of which CXCL8/IL-8 was one of the highest loading variables ( $\mathrm{r}=0.302$; online supplementary tables S4 and S5). Other top variables for biomarker factor 8 were the anti-inflammatory markers serum $\mathrm{IL}-10(\mathrm{r}=-0.440)$ and tumour necrosis factor $(\mathrm{r}=0.336)$. Overall, the network analysis suggests a significant interaction between specific microbiota groups and human biomarkers associated with inflammation.

We also performed a CCA to test the association of clinical variables with variation in microbial composition at different taxonomic levels. To account for multiple measures per subject, we limited our analysis to the initial samples collected from group I subjects. Both serum MMP-7 and the percentage of sputum neutrophils had a significant correlation with the microbial composition at the OTU level ( $p<0.05$ using CCA; table 4). Sputum CXCL8/IL-8, the percentage of sputum neutrophils and serum colony stimulating factor-2 were significantly associated with variation at the phylum level, while sputum CXCL8/IL-8, the percentage of sputum neutrophils and serum MMP-7 were significant at the genus level ( $\mathrm{p}<0.05$ using CCA; table 4$)$. 


\section{Discussion}

COPD occurrence and severity are mediated through complex interactions between the host immune system, environmental factors and respiratory pathogens. Our study adds further insights into the role of the lung microbiome in COPD with the inclusion of a large patient cohort and repeated longitudinal sampling over multiple clinical visits. A key finding is the association of changes in the lung microbiome
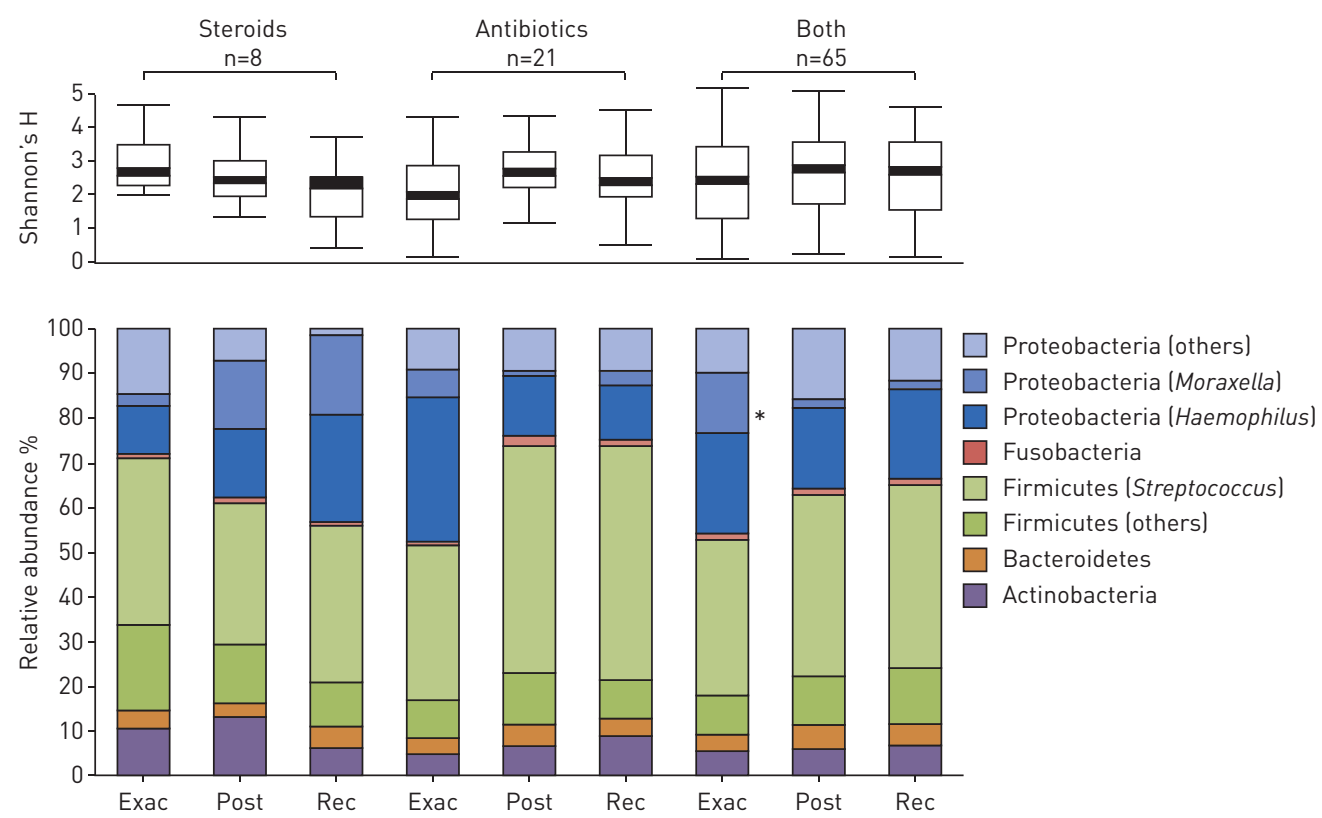

FIGURE 4 Microbiome changes due to oral corticosteroids and antibiotics. $\alpha$ diversity (Shannon's H) and composition of major taxonomic groups at both phylum and genus levels in exacerbations (Exac), post-therapy (Post) and recovery $(\operatorname{Rec})$ samples of subjects treated with steroids, antibiotics or a combination of both. Only visit series with a complete cycle of exacerbation, post-therapy and recovery visits were included. The number of visit series is indicated for each subgroup. *: $p<0.05$ using ANOVA.

a)

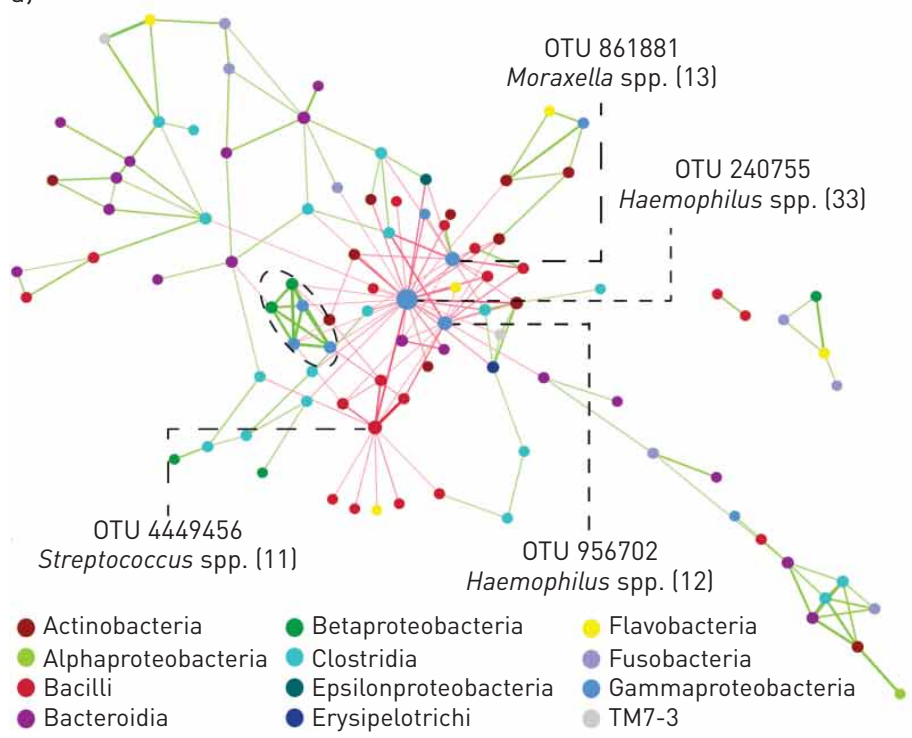

b)

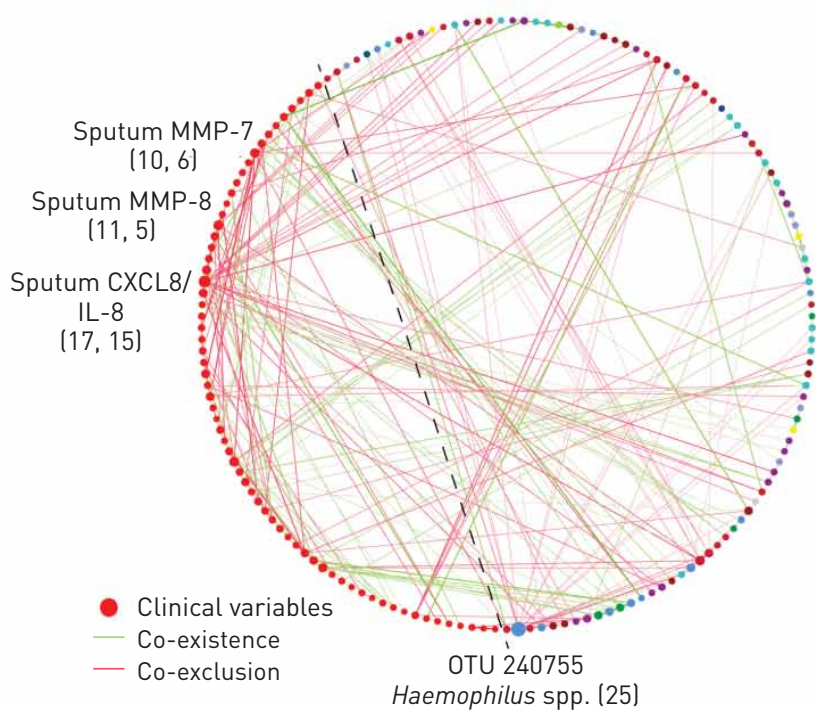

FIGURE 5 Bacterial co-existence and co-exclusion relationships with operational taxonomic units (OTUs) and host factors. Interaction networks of a) microbiome and b) microbiome and clinical factors. Each node represents an OTU or a clinical trait. The OTUs were coloured by the class-level taxonomy. The five co-existence OTUs are highlighted in the dotted ellipse in a). The clinical traits were grouped together at the left of the network in b). Each edge represents a significant correlation coloured by co-existence or co-exclusion relationships. Edge width is proportional to the absolute value of Pearson correlation coefficient. The size of the node is proportional to its degree of connectivity. The degrees are shown in parentheses for highly connected nodes. For the clinical variables, both the total degree and the degree of connectivity to OTUs are shown. MMP: matrix metalloproteinase; CXCL: chemokine (C-X-C motif) ligand; IL: interleukin. 
TABLE 4 List of clinical variables significantly associated with microbial $\alpha$ and $\beta$ diversity in group I subjects

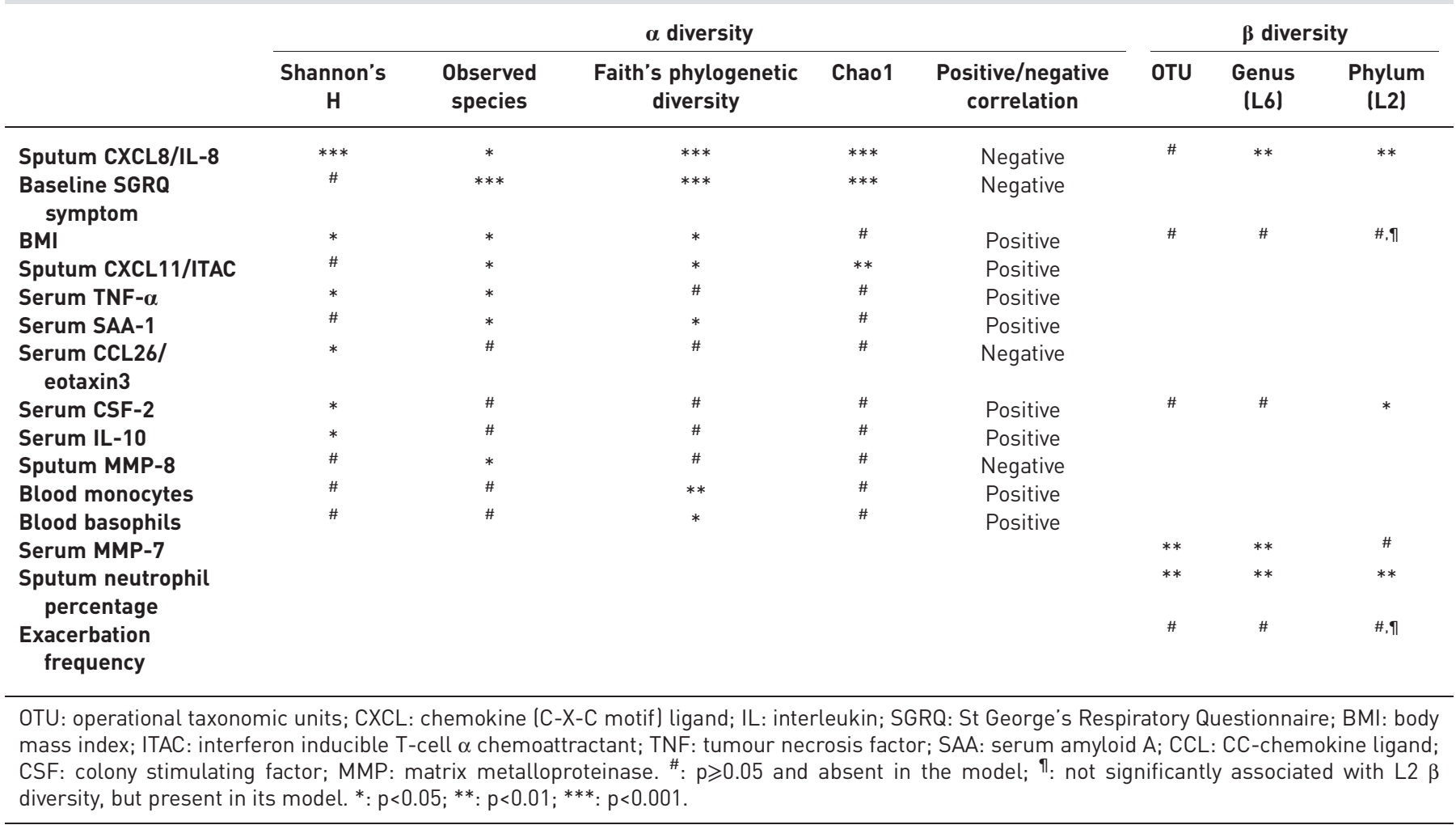

with multiple characteristics of COPD, including specific exacerbation phenotypes, treatment regimen and the levels of key sputum and serum mediators.

In agreement with previous studies, COPD exacerbation events appear to be associated with decreased microbial diversity and increased proportion of Proteobacteria $[8,23]$. In addition, there was a remarkable proliferation of Moraxella in a subgroup of subjects during exacerbations (36 out of 87 subjects). $M$. catarrhalis is a critical respiratory pathogen that enhances airway inflammation by stimulating several neutrophil-related components during COPD exacerbations [21]. Consistently, there was a significant positive correlation between the abundance of Moraxella and the percentage of sputum neutrophils. Despite the heterogeneous nature of COPD, our results suggest that specific subgroups of COPD subjects are particularly susceptible to alternation of microbiome during exacerbations.

Distinct microbial populations existed during exacerbations in subjects with bacterial and eosinophilic exacerbations, which were more dissimilar from each other in microbiome profiles than either was from viral exacerbations. This is in agreement with the suggestion that bacterial and eosinophilic exacerbations reflect fundamental differences in their immunopathogenesis, whereas virus exacerbations are often associated with both bacterial infections and increase of eosinophils [12, 24]. The presence or absence of eosinophilic inflammation could be a potential biomarker for stratification of the underlying associated microbiome.

Our study shows that current standard-of-care treatments potentially alter the lung microbiome. In particular, a reduction in microbial diversity and an increased Proteobacteria:Firmicutes ratio toward recovery were observed in subjects treated with steroids alone, whereas the trend was reversed in subjects who received antibiotics. Similar changes in the lung microbiome after these treatments were observed in a smaller study of 12 COPD subjects [8]. In support of reports on the limited efficacy and greater side-effects of steroids $[25,26]$ our results suggest that steroids alone could affect the lung microbiome and underscore the importance of patient stratification approaches, such as blood eosinophil-guided prednisolone therapy [13], toward more precise drug management strategies.

In our network analysis, several bacterial OTUs, in particular the OTU 240755 belonging to Haemophilus, were identified as microbial "hubs" that had a disproportionately large number of negative connections with other OTUs. Such correlations were highly robust across all samples, indicating that this might represent a general pattern of the COPD lung microbiome. Overgrowth of these bacterial OTUs could thus 
drive respiratory tract dysbiosis, which has been suggested to be a potential cause of lung disease exacerbations [27]. Recent studies have highlighted the importance of ecological interactions in multiple human body habitats. An emerging paradigm is the "keystone species" hypothesis, where even marginal changes in the abundances of relatively few bacterial species could have profound effects on the overall microbial community structure and consequently alter human disease states [22, 28, 29]. Based on our network analyses we speculate that increased abundance of Haemophilus spp., and possibly other Proteobacteria, might remodel the normal lung microbial ecosystem into a state of dysbiosis which could elicit a host pro- inflammatory response. Our results suggest that the "keystone species" hypothesis in context of the lung microbiome warrants further study, since it might provide a conceptual basis for novel therapeutic strategies that target a few key bacterial targets to counteract a dysbiotic microbial community in COPD.

Sputum CXCL8/IL-8 was significantly associated with both lung microbiome diversity and its overall community structure, thus CXCL8/IL-8 could be a suitable biomarker to monitor the overall lung microbial population. Sputum CXCL8/IL-8 has long been recognised to play a prominent role in COPD $[30,31]$. It induces airway inflammation by predominantly recruiting neutrophils and upregulating airway mucin genes, resulting in mucus production [32]. Elevated sputum CXCL8/IL-8 levels are associated with elevated COPD severity [30, 33, 34]. Pathogenic members of the genera Haemophilus and Moraxella are able to directly induce inflammation by exposing the host to lipopolysaccharides and other pathogen-associated molecular patterns [35]. Interestingly, our network analysis revealed that multiple members in the lung microbiome were negatively correlated with sputum CXCL8/IL-8, and several OTUs comprised of Haemophilus, Moraxella and Streptococcus species. Potentially, these pathogens could also indirectly trigger the excessive production of CXCL8/IL-8 through dysbiosis of the lung microbiota. Therefore, we speculate that the lung microbiome could serve as an additional line of defence that shapes the lung inflammatory response induced by respiratory pathogens [27].

There are several caveats to our study. First, despite a large cohort size, our survey focused exclusively on COPD patients with exacerbations, with no healthy or nonexacerbator control subjects. Data from these populations could be informative in defining the normal lung microbiota as well as the changes of microbial composition resulting in COPD onset. Second, besides bacteria, the importance of viruses and fungi in COPD is just beginning to be appreciated and studied [36-38]. A systems biology view of bacterial, viral and fungal microbiomes integrating additional host response factors such as host transcriptome and metabolome profiles would boost our understanding of the host-microbiota interaction and its implication in disease aetiology. Finally, our results need to be replicated in further larger and distinct patient populations, including those with different ethnicity and biogeographical backgrounds.

In conclusion, we show that changes in the lung microbiome are associated with COPD exacerbation events and are potentially implicated in mediating host inflammatory responses in some subjects. Moreover, this work furthers our understanding of the lung microbiome in COPD and opens potential avenues for new biomarkers and respiratory therapeutics.

\section{Acknowledgements}

We thank Deepali Jhamb and Michal Magid-Slav (Computational Biology, GSK R\&D, King of Prussia, PA, USA) for their advice on statistical analyses of this study.

\section{References}

1 Lopez $\mathrm{AD}$, Shibuya $\mathrm{K}$, Rao $\mathrm{C}$, et al. Chronic obstructive pulmonary disease: current burden and future projections. Eur Respir J 2006; 27: 397-412.

2 Franklin W, Lowell FC, Michelson AL, et al. Chronic obstructive pulmonary emphysema; a disease of smokers. Ann Intern Med 1956; 45: 268-274.

3 Taraseviciene-Stewart L, Douglas IS, Nana-Sinkam PS, et al. Is alveolar destruction and emphysema in chronic obstructive pulmonary disease an immune disease? Proc Am Thorac Soc 2006; 3: 687-690.

4 Miravitlles M, Espinosa C, Fernández-Laso E, et al. Relationship between bacterial flora in sputum and functional impairment in patients with acute exacerbations of COPD. Study Group of Bacterial Infection in COPD. Chest 1999; 116: 40-46.

5 Ball P. Epidemiology and treatment of chronic bronchitis and its exacerbations. Chest 1995; 108: Suppl. 2, 43S-52S.

6 Soler N, Torres A, Ewig S, et al. Bronchial microbial patterns in severe exacerbations of chronic obstructive pulmonary disease (COPD) requiring mechanical ventilation. Am J Respir Crit Care Med 1998; 157: 1498-1505.

7 Monsó E, Ruiz J, Rosell A, et al. Bacterial infection in chronic obstructive pulmonary disease. A study of stable and exacerbated outpatients using the protected specimen brush. Am J Respir Crit Care Med 1995; 152: $1316-1320$.

8 Huang YJ, Sethi S, Murphy T, et al. Airway microbiome dynamics in exacerbations of chronic obstructive pulmonary disease. J Clin Microbiol 2014; 52: 2813-2823.

9 Molyneaux PL, Mallia P, Cox MJ, et al. Outgrowth of the bacterial airway microbiome following rhinovirus exacerbation of chronic obstructive pulmonary disease. Am J Respir Crit Care Med 2013; 188: 1224-1231. 

infections by use of $16 \mathrm{~s}$ ribosomal DNA terminal restriction fragment length polymorphism profiling. $J$ Clin Microbiol 2004; 42: 5176-5183.

12 Bafadhel M, McKenna S, Terry S, et al. Acute exacerbations of chronic obstructive pulmonary disease: identification of biologic clusters and their biomarkers. Am J Respir Crit Care Med 2011; 184: 662-671.

for chronic obstructive pulmonary disease: a randomized placebo-controlled trial. Am J Respir Crit Care Med 2012; 186: 48-55

14 Anthonisen NR, Manfreda J, Warren CP, et al. Antibiotic therapy in exacerbations of chronic obstructive pulmonary disease. Ann Intern Med 1987; 106: 196-204.

15 Rodriguez-Roisin R. Toward a consensus definition for COPD exacerbations. Chest 2000; 117: Suppl. 2, 398S-401S.

16 National Institute for Health and Clinical Excellence. Chronic Obstructive Pulmonary Disease in Over 16s: Diagnosis and Management. London, National Institute for Health and Clinical Excellence, 2010.

17 Caporaso JG, Kuczynski J, Stombaugh J, et al. QIIME allows analysis of high-throughput community sequencing data. Nat Methods 2010; 7: 335-336.

18 Benjamini Y, Hochberg Y. Controlling the false discovery rate: a practical and powerful approach for multiple testing. J R Statist Soc B 1995; 57: 289-300.

19 Erkan L, Uzun O, Findik S, et al. Role of bacteria in acute exacerbations of chronic obstructive pulmonary disease. Int I Chron Obstruct Pulmon Dis 2008; 3: 463-467.

20 Sethi S, Murphy TF. Bacterial infection in chronic obstructive pulmonary disease in 2000: a state-of-the-art review. Clin Microbiol Rev 2001; 14: 336-363.

21 Parameswaran GI, Wrona CT, Murphy TF, et al. Moraxella catarrhalis acquisition, airway inflammation and protease-antiprotease balance in chronic obstructive pulmonary disease. BMC Infect Dis 2009; 9: 178.

22 Faust K, Sathirapongsasuti JF, Izard J, et al. Microbial co-occurrence relationships in the human microbiome. PLoS Comput Biol 2012; 8: e1002606.

23 Millares L, Ferrari R, Gallego M, et al. Bronchial microbiome of severe COPD patients colonised by Pseudomonas aeruginosa. Eur J Clin Microbiol Infect Dis 2014; 33: 1101-1111.

24 Bathoorn E, Kerstjens H, Postma D, et al. Airways inflammation and treatment during acute exacerbations of COPD. Int J Chron Obstruct Pulmon Dis 2008; 3: 217-229.

25 Leuppi JD, Schuetz P, Bingisser R, et al. Short-term vs conventional glucocorticoid therapy in acute exacerbations of chronic obstructive pulmonary disease: the REDUCE randomized clinical trial. JAMA 2013; 309: 2223-2231.

26 McEvoy CE, Niewoehner DE. Adverse effects of corticosteroid therapy for COPD. A critical review. Chest 1997; 111: 732-743.

27 Dickson RP, Martinez FJ, Huffnagle GB. The role of the microbiome in exacerbations of chronic lung diseases. Lancet 2014; 384: 691-702.

28 Fisher CK, Mehta P. Identifying keystone species in the human gut microbiome from metagenomic timeseries using sparse linear regression. PLoS One 2014; 9: e102451.

29 Hajishengallis G, Darveau RP, Curtis MA. The keystone-pathogen hypothesis. Nat Rev Microbiol 2012; 10: 717-725.

30 Larsson K. Inflammatory markers in COPD. Clin Respir J 2008; 2: Suppl 1, 84-87.

31 Chung KF. Cytokines in chronic obstructive pulmonary disease. Eur Respir J 2001; 34: Suppl. 50s-59s.

32 Bautista MV, Chen Y, Ivanova VS, et al. IL-8 regulates mucin gene expression at the posttranscriptional level in lung epithelial cells. J Immunol 2009; 183: 2159-2166.

33 Zhang $\mathrm{X}$, Zheng $\mathrm{H}$, Zhang $\mathrm{H}$, et al. Increased interleukin (IL)-8 and decreased IL-17 production in chronic obstructive pulmonary disease (COPD) provoked by cigarette smoke. Cytokine 2011; 56: 717-725.

34 Bhowmik A, Seemungal TA, Sapsford RJ, et al. Relation of sputum inflammatory markers to symptoms and lung function changes in COPD exacerbations. Thorax 2000; 55: 114-120.

35 Ishii KJ, Koyama S, Nakagawa A, et al. Host innate immune receptors and beyond: making sense of microbial infections. Cell Host Microbe 2008; 3: 352-363.

36 Kistler A, Avila PC, Rouskin S, et al. Pan-viral screening of respiratory tract infections in adults with and without asthma reveals unexpected human coronavirus and human rhinovirus diversity. J Infect Dis 2007; 196 : 817-825.

37 Cui L, Lucht L, Tipton L, et al. Topographic diversity of the respiratory tract mycobiome and alteration in HIV and lung disease. Am J Respir Crit Care Med 2015; 191: 932-942.

38 Ghannoum MA, Jurevic RJ, Mukherjee PK, et al. Characterization of the oral fungal microbiome (mycobiome) in healthy individuals. PLoS Pathog 2010; 6: e1000713. 